\title{
Screening for genetic abnormalities involved in ovarian carcinogenesis using retroviral expression libraries
}

\author{
TOMOAKI WADA $^{1,2}$, YOSHIHIRO YAMASHITA ${ }^{1}$, YASUSHI SAGA ${ }^{2}$, KAYOKO TAKAHASHI $^{2}$, \\ KOJI KOINUMA ${ }^{1}$, YOUNG LIM CHOI ${ }^{1}$, RURI KANEDA ${ }^{1}$, SHIN-ICHIRO FUJIWARA ${ }^{1}$, \\ MANABU SODA $^{1}$, HIDEKI WATANABE ${ }^{1}$, KENTARO KURASHINA $^{1}$, HISASHI HATANAKA ${ }^{1}$, \\ MUNEHIRO ENOMOTO $^{1}$, SHUJI TAKADA ${ }^{1}$, HIROYUKI MANO ${ }^{1,3}$ and MITSUAKI SUZUKI ${ }^{2}$ \\ ${ }^{1}$ Division of Functional Genomics and ${ }^{2}$ Department of Obstetrics and Gynecology, School of Medicine, \\ Jichi Medical University, Tochigi; ${ }^{3}$ CREST, Japan Science and Technology Agency, Saitama, Japan
}

Received April 24, 2009; Accepted June 23, 2009

DOI: 10.3892/ijo_00000410

\begin{abstract}
The purpose of this study was to screen for genes involved in ovarian carcinogenesis in an attempt to develop an effective molecular-targeted therapy for ovarian cancer. We constructed retroviral expression libraries for the human ovarian cancer cell lines SHIN-3 and TYK-CPr, and performed a focus formation assay with $3 \mathrm{~T} 3$ cells. As a result, proteasome subunit beta-type 2 (PSMB2), ubiquitin-specific protease 14 (USP14), and keratin 8 (KRT8) were identified from SHIN-3, and polymerase II RNA subunit (POLR2E), chaperonin containing T-complex polypeptide 1 subunit 4 (CCT4), glia maturation factor beta $(G M F B)$, and neuroblastoma ras viral oncogene homolog (NRAS) from TYK-CPr. NRAS gene analysis revealed a $\mathrm{CAA} \rightarrow \mathrm{AAA}$ substitution at codon 61 , resulting in a Glu $\rightarrow$ Lys change at position 61 . When the mutant $N R A S$ was introduced into fibroblasts for its expression, many transformed foci were generated, confirming the transforming ability of the mutant NRAS.
\end{abstract}

\section{Introduction}

Ovarian cancer is the fifth most common cause of death from gynecologic malignancies in the USA. Approximately 25,000 women are affected by ovarian cancer every year, and about 14,000 die of this disease (1). In recent years, debulking surgery followed by multidrug therapy with platinum and taxine drugs have been used, with some improvement in prognosis (2); however, the 5-year survival rate remains at

Correspondence to: Dr Yasushi Saga, Department of Obstetrics and Gynecology, School of Medicine, Jichi Medical university, 3311-1 Yakushiji, Shimotsuke, Tochigi 329-0498, Japan

E-mail: saga@jichi.ac.jp

Key words: ovarian cancer, oncogene, retroviral expression screening, NRAS about $50 \%$ (3). This is due to the lack of effective therapy for treatment-resistant or recurrent ovarian cancer.

A series of recent studies have reported that STI571, which targets the $B C R-A B L$ gene responsible for chronic myelogenous leukemia, is effective for this disease, and that the anti-CD20 antibody rituximab is highly effective for B-cell lymphocytic leukemia (4). These observations have demonstrated that it is clinically very important to elucidate the pathogenesis of malignancies and thereby develop molecular-targeted therapy for them. To improve the prognosis of patients with ovarian cancer, it is important to define genetic abnormalities involved in the onset of this disease, and to develop effective molecular-targeted therapies for ovarian cancer.

To date, several studies have reported that mutations in the $p 53$ gene (5) or deletions in the BRCAI and BRCA2 genes (6) are common in ovarian cancer. However, there is no evidence that these genetic abnormalities are directly involved in the development of ovarian cancer.

In a recent study, we constructed a full-length cDNA expression library of non-small cell lung cancer (NSCLC) using a retroviral vector, and demonstrated by functional screening that a fusion gene, composed of portions of the echinoderm microtubule-associated protein-like 4 (EML4) and anaplastic lymphoma kinase $(A L K)$ genes, was involved in the development of NSCLC (7). In this study, we aimed to screen for genes responsible for ovarian carcinogenesis using similar techniques.

\section{Materials and methods}

Cell culture. Ovarian serous cystadenocarcinoma cell line SHIN-3 (8) and ovarian undifferentiated carcinoma cell line TYK-CPr (JCRB0234.1, Health Science Research Resources Bank: HSRRB, Osaka, Japan) (9) cells were cultured in Dulbecco's modified Eagle's medium/F12 (DMEM/F12; Invitrogen, Carlsbad, CA, USA) medium supplemented with $10 \%$ fetal bovine serum (Invitrogen), $2 \mathrm{mM}$ L-glutamine (Invitrogen), and 1\% penicillin-streptomycin (Invitrogen). The BOSC23 packaging cell line for ecotropic retroviruses (10) and mouse $3 \mathrm{~T} 3$ fibroblasts (American Type Culture 
Collection: ATCC) were maintained in DMEM/F12 supplemented with $10 \%$ fetal bovine serum and $2 \mathrm{mM}$ L-glutamine, and $1 \%$ penicillin-streptomycin.

Construction of a retrovirus library. Total RNA was extracted from SHIN-3 and TYK-CPr cells with the use of an RNeasy Mini column and RNase-free DNase (Qiagen, Valencia, CA, USA), and first-strand cDNA was synthesized from the RNA with PowerScript reverse transcriptase, a SMART IIA oligonucleotide, and CDS primer IIA (Clontech, Palo Alto, CA, USA). The resulting cDNA molecules were then amplified for 15 cycles with the 5'-PCR primer IIA and a SMART PCR cDNA synthesis kit (Clontech), with the exception that LA Taq polymerase (Takara Bio, Shiga, Japan) was substituted for the Advantage 2 DNA polymerase provided with the kit. The PCR products were treated with proteinase $\mathrm{K}$, rendered blunt-ended with T4 DNA polymerase, and ligated to a $B s t X I$ adapter (Invitrogen). Unbound adapters were removed with a cDNA size fractionation column (Invitrogen), and the modified cDNAs were ligated into the pMX retroviral plasmid (11) that had been digested with BstXI. The pMX-cDNA plasmids were introduced into ElecroMax DH10B cells (Invitrogen) by electroporation.

Focus formation assay. BOSC23 cells $\left(1.8 \times 10^{6}\right)$ were seeded onto $6-\mathrm{cm}$ culture plates, cultured for 1 day, and then transfected with a mixture comprising $2 \mu \mathrm{g}$ of retroviral plasmids, $0.5 \mu \mathrm{g}$ of pGP plasmid (Takara Bio), $0.5 \mu \mathrm{g}$ of pE-eco plasmid (Takara Bio), and $18 \mu 1$ of Lipofectamine reagent (Invitrogen). Two days after transfection, polybrene (Sigma, St. Louis, MO, USA) was added at a concentration of $4 \mu \mathrm{g} / \mathrm{ml}$ to the culture supernatant, which was then used to infect $3 \mathrm{~T} 3$ cells for $48 \mathrm{~h}$. For the focus formation assay, the culture medium of $3 \mathrm{~T} 3$ cells was changed to DMEM-high glucose (Invitrogen) supplemented with $5 \%$ calf serum and $2 \mathrm{mM}$ L-glutamine. Transformed foci were isolated after 3 weeks of culture.

Recovery of cDNAs from $3 T 3$ cells. Each $3 \mathrm{~T} 3$ cell clone was harvested with a cloning syringe and cultured independently in a $10-\mathrm{cm}$ culture plate. Genomic DNA was subsequently extracted from the cells and subjected to PCR with the 5'-PCR primer IIA and LA Taq polymerase for 50 cycles of $98^{\circ} \mathrm{C}$ for $20 \mathrm{sec}$ and $68^{\circ} \mathrm{C}$ for $6 \mathrm{~min}$. Amplified genomic fragments were purified by gel electrophoresis and ligated into the pT7Blue-2 vector (EMD Biosciences, San Diego, CA, USA) for nucleotide sequencing. The cDNAs obtained were introduced into the pMXS plasmid to prepare a recombinant retrovirus, which was used again to test its 3T3-transforming ability.

Analysis of the NRAS gene in the TYK-CPr cell line. Using as the substrate the cDNA that had been synthesized to construct the retroviral library, along with NRAS primers [5' primer (GTGGAGCTTGAGGTTCTTGC) and 3' primer (GCAG CTTGAAAGTGGCTCTT)], the NRAS gene was amplified by PCR for 30 cycles of $98^{\circ} \mathrm{C}$ for $30 \mathrm{sec}, 62^{\circ} \mathrm{C}$ for $30 \mathrm{sec}$, and $68^{\circ} \mathrm{C}$ for $30 \mathrm{sec}$. The amplified DNA fragments were separated by electrophoresis, then purified, and inserted into the pGEM-T Easy Vector (Promega Corp., Madison, WI, USA) for sequencing.

\section{Results}

Construction of full-length cDNA expression libraries for SHIN-3 and TYK-CPr cells. cDNAs from SHIN-3 and TYK-CPr cells were inserted into the pMXS retroviral plasmid, which was then introduced into DH10B cells by electroporation. As a result, we obtained plasmid libraries of cDNA clones from SHIN-3 (1.1 $\times 10^{6}$ colony-forming units, or cfu) and TYK-CPr $\left(1.2 \times 10^{6} \mathrm{cfu}\right)$ cells. From each of these plasmid clone libraries, 24 clones were picked up at random. In addition, to ascertain whether the cDNA inserts were full-length (complete reading frame), 10 clones were selected from each library, and $~ 500$ bp of both ends of the cDNA insert were sequenced. The identified sequences were compared with the University of California-Santa Cruz Genome Browser Database (http://genome.ucsc.edu) by BLAST search (12). As a result, 7 of the 10 TYK-CPr-derived clones and 7 of the 10 SHIN-3-derived clones contained full-length cDNA inserts (data not shown). We therefore concluded that the retroviral cDNA expression libraries were of sufficient complexity and adequately enriched in fulllength cDNAs for the present study.

Screening for transformed clones. 3T3 cells were transfected with retroviral cDNA expression libraries, and, after 3 weeks of culture, transformed clones were isolated. As a result, 17 and 15 transformed clones were identified for TYK-CPr and SHIN-3, respectively (Fig. 1).

Transformed clones were isolated using a cloning syringe, and cultured in separate dishes to extract genomic DNA from each clone. The genomic DNA was amplified by PCR using the same primers as those employed in cDNA amplification for library construction. As shown in Fig. 2, a single sharp cDNA band per clone was identified in about half of the clones.

Analysis of cDNAs recovered from transformed cells. Transformed clones, whose genomic DNA amplified by PCR gave a single sharp cDNA band, were selected, and their respective cDNAs were sequenced for gene identification. As a result, proteasome subunit beta-type 2 (PSMB2), ubiquitin-specific protease 14 (USP14), and keratin 8 (KRT8) were identified from SHIN-3, and polymerase II RNA subunit (POLR2E), chaperonin containing $T$-complex polypeptide 1 subunit 4 (CCT4), glia maturation factor beta (GMFB), and neuroblastoma ras viral oncogene homolog (NRAS) from TYK-CPr. All cDNAs contained complete open reading frames (ORF).

Analysis of the NRAS gene in the TYK-CPr cell line. Focusing on NRAS among the genes identified in this study, we examined the TYK-CPr-derived cDNA for point mutations, and found a codon 61 mutation $(\mathrm{CAA} \rightarrow \mathrm{AAA}, \mathrm{Glu} \rightarrow \mathrm{Lys})$ (Fig. 3).

Furthermore, the resulting mutant NRAS (NRAS ${ }^{\mathrm{Q} 61 \mathrm{~K}}$ ) cDNA was inserted into the pMX plasmid to construct a recombinant retrovirus, which was transfected into $3 \mathrm{~T} 3$ cells for a focus formation assay. As shown in Fig. 4, the identified NRAS ${ }^{\mathrm{Q} 61 \mathrm{~K}}$ generated many transformed foci, confirming its transforming ability. 


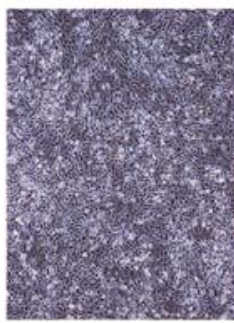

pMXS

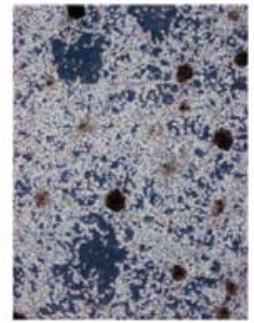

PMXS-vRAS

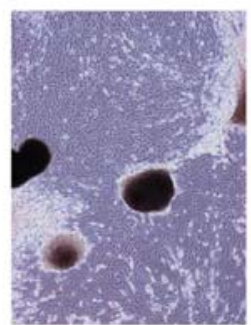

pMXS-cDNA
Figure 1. Focus formation assay with a retroviral library derived from TYK-CPr cells. Mouse 3T3 cells were infected with the empty virus (pMXS), a retrovirus expressing v-Ras as a positive control (pMXS-vRAS), or retroviruses from the TYK-CPr cell library (pMXS-cDNA). The cultures were photographed 3 weeks after infection.

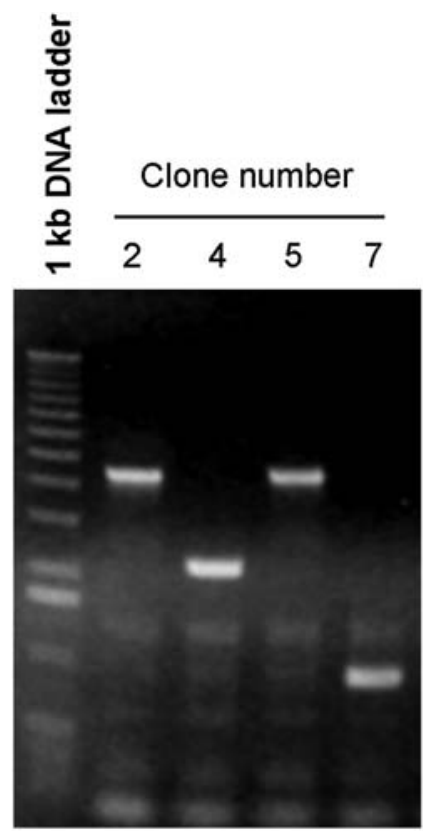

Figure 2. Genomic DNA isolated from transformed 3T3 cell foci (clone numbers 2, 4, 5, and 7) was subjected to PCR for amplification of the DNA inserts. The left lane contains DNA size markers (1-kbp DNA ladder; Invitrogen).

$\begin{array}{llllllllllll}57 & D & T & A & G & Q & E & E & Y & S & A & M\end{array}$ gatacagctggacaagaagagtacagtgccatg Published

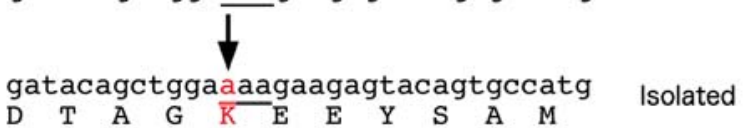

Figure 3. The amino acid sequence in the vicinity of the amino acid residue at position 61 of NRAS protein and the corresponding NRAS cDNA (NM_002524) codon sequence are shown in the upper row (published) Similarly, the amino acid sequence and the corresponding NRAS cDNA (isolated) codon sequence identified in this study are shown in the lower row. In the screened cDNA, the glutamine-encoding codon (caa) at position 61 had been converted to the lysine-encoding codon (aaa). The site of the nucleotide substitution is indicated in red.

To confirm that the NRAS ${ }^{\mathrm{Q} 1 \mathrm{~K}}$ mutation did not arise during the process of library construction or PCR, we sequenced the NRAS gene in 10 randomly selected TYK-CPr cell

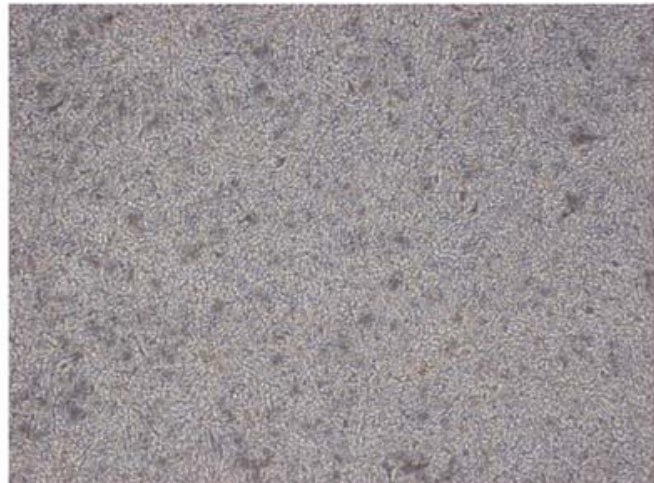

Figure 4. A recombinant retrovirus encoding NRAS ${ }^{\mathrm{Q} 61 \mathrm{~K}}$ was used to infect $3 \mathrm{~T} 3$ cells. The cells were photographed after culture for 2 weeks.

clones, and found a CAA $\rightarrow$ AAA substitution at codon 61 in 7 clones. This indicates that the TYK-CPr cell line has a wild-type allele and a mutant allele (NRAS $\left.{ }^{\mathrm{Q} 61 \mathrm{~K}}\right)$.

\section{Discussion}

The focus formation assay in mouse 3T3 fibroblasts has been widely used to identify oncogenes (13). The conventional 3T3 focus formation assay involves the introduction of cancer cell-derived genomic DNA, followed by screening for focus-forming, transformed clones of $3 \mathrm{~T} 3$ cells. To date, many oncogenes such as $R A S, A B L$, and $R A F$ have been identified using this assay. However, the assay involving the introduction of genomic DNA alone has the major disadvantage of a lower screening ability, that is, the expression of oncogenes is controlled by their own enhancer/promoter region. However, the enhancer/promoter region of oncogenes functioning in ovarian cancer is not necessarily active in $3 \mathrm{~T} 3$ fibroblasts. Therefore, the possibility of the successful identification of ovarian cancer-related oncogenes by the classical focus formation assay involving the introduction of genomic DNA alone is small. To ensure that all genes introduced into 3T3 cells are expressed at sufficient levels, it is necessary that their transcription be regulated by exogenous promoters and enhancers.

The retroviral vector is a type of vector most commonly used for gene introduction, and has advantages in that the cDNA inserted between the left and right long terminal repeats (LTR) is integrated directly into the chromosomes of infected cells, and viral vectors can be produced at high titers using packaging cells $(14,15)$.

In this study, to screen for genes involved in ovarian carcinogenesis, we attempted to construct cDNA-expressing recombinant retroviral libraries that were engineered to express cDNA from retroviral LTR, and succeeded in constructing libraries with a sufficient complexity and mean insert size, derived from the ovarian cancer cell lines SHIN-3 and TYK-CPr. Using these libraries, we performed a focus formation assay in $3 \mathrm{~T} 3$ cells, and were able to recover the cDNA inserts easily from the transformed clones employing the primers used for cDNA synthesis. From each of 7 clones, a single cDNA was identified and sequenced. As a result, $P S M B 2, U S P 14$, and KRT8 were screened from SHIN-3 cells, and POLR2E, CCT4, GMFB, and NRAS from TYK-CPr 
cells. Focusing on NRAS, known as an oncogene, we analyzed it, and identified a $\mathrm{CAA} \rightarrow \mathrm{AAA}$ substitution at codon 61 , resulting in a Glu $\rightarrow$ Lys change at position 61 . When the mutant NRAS was transfected into fibroblasts for its expression, many transformed foci were generated, confirming the transforming ability of the mutant NRAS.

The RAS gene family is a group of three oncogenes, KRAS, HRAS, and NRAS, which are most commonly activated in human malignant neoplasms (16). For RAS activation, an amino acid substitution at position 12, 13, 59, or 61 is important. It was reported in thyroid tumors that a $\mathrm{CAA} \rightarrow \mathrm{CGA}$ substitution at codon 61 of NRAS resulted in a $\mathrm{Glu} \rightarrow$ Arg change at position 61 and NRAS activation (17). It was also noted in the neuroblastoma cell line SK-N-SH that a $\mathrm{Glu} \rightarrow$ Lys mutation at position 61 of NRAS resulted in its activation (18).

Furthermore, KRAS mutations were reported to occur in about $50 \%$ of ovarian mucinous carcinomas $(19,20)$ and $30 \%$ of serous borderline ovarian tumors (21). In addition, HRAS mutations were reported in about $6 \%$ of ovarian cancers (22). However, no NRAS activation in ovarian cancers has been reported to date (22). Since the cell proliferation function of the HRAS, KRAS, and NRAS gene products overlaps with one another in many cases, the reason for selective KRAS activation in specific histological types of ovarian cancer is unclear.

This study is the first in the world to identify NRAS gene activation by point mutation in an ovarian cancer cell line. However, no NRAS gene activation has been reported in ovarian cancer patients. In the future, it will be necessary to analyze clinical samples for NRAS mutations, particularly amino acid substitutions at position 61 . TYK-CPr, in which an activated NRAS gene was identified in this study, is a cell line derived from an undifferentiated ovarian cancer with a clinically poor prognosis. This suggests that NRAS activation is associated with specific histological types of ovarian cancer.

Recently, sorafenib, a molecular-targeted therapeutic drug targeting activated RAS, has been developed and used clinically. The drug inhibits Raf kinase downstream of RAS, thereby blocking the RAS/MEK/ERK signaling pathway and exerting antitumor effects (23). Such a molecular-targeted drug may be effective for ovarian cancer patients with the NRAS mutations reported here.

Among the genes screened in this study, proteasome has been reported to be involved in cell cycle regulation and apoptosis (24). In addition, keratin 8 has been reported to be involved in malignant transformation and cancer cell invasion (25). These genes may be ovarian cancer-related oncogenes, although further studies are needed.

\section{References}

1. Jemal A, Siegel R and Ward E: Cancer statistics, 2008. CA Cancer J Clin 58: 71-76, 2008.

2. Armstrong DK, Bundy B and Wenzel L: Intraperitoneal cisplatin and paclitaxel in ovarian cancer. N Engl J Med 354: 34-43, 2006.
3. Druker BJ, Talpaz M and Resta DJ: Efficacy and safety of a specific inhibitor of the BCR-ABL tyrosine kinase in chronic myeloid leukemia. N Engl J Med 344: 1031-1037, 2001.

4. Maloney DG, Grillo-Lopez AJ and White CA: IDEC-C2B8 (Rituximab) anti-CD20 monoclonal antibody therapy in patients with relapsed low-grade non-Hodgkin's lymphoma. Blood 90: 2188-2195, 1997.

5. Okamoto A, Sameshima Y and Yokoyama S: Frequent allelic losses and mutations of the p53 gene in human ovarian cancer. Cancer Res 51: 5171-5176, 1991.

6. Esteller M, Silva JM and Dominguez G: Promoter hypermethylation and BRCA1 inactivation in sporadic breast and ovarian tumors. J Natl Cancer Inst 92: 564-569, 2000.

7. Soda M, Choi YL and Enomoto M: Identification of the transforming EML4-ALK fusion gene in non-small-cell lung cancer. Nature 448: 545-546, 2007.

8. Imai S, Kiyozuka Y and Maeda H: Establishment and characterization of a human ovarian serous cystadenocarcinoma cell line that produces the tumor markers CA-125 and tissue polypeptide antigen. Oncology 47: 177-184, 1990.

9. Yoshizawa H, Adashi S and Misawa Y: Isolation of cisplatinresistant subline from human ovarian cancer cell line and analysis of its cell-biological characteristics (in Japanese). Nihon Sanfujinka Gakkai-shi 141: 7-14, 1989.

10. Pear WS, Nolan GP and Scott ML: Production of high-titer helper-free retroviruses by transient transfection. Proc Natl Acad Sci USA 90: 8392-8396, 1993.

11. Onishi M, Kinoshita S and Morikawa Y: Applications of retrovirus-mediated expression cloning. Exp Hematol 24: 324-329, 1996.

12. Kent WJ: BLAT - the BLAST-like alignment tool. Genome Res 12: 656-664, 2002.

13. Aaronson SA: Growth factors and cancer. Science 254: 1146-1153, 1991.

14. Kisanuki H, Choi YL and Wada T: Retroviral expression screening of oncogenes in pancreatic ductal carcinoma. Eur J Cancer 41: 2170-2175, 2005.

15. Choi YL, Moriuchi R and Osawa M: Retroviral expression screening of oncogenes in natural killer cell leukemia. Leuk Res 29: 943-949, 2005.

16. Shih TY and Weeks MO: Oncogenes and cancer: the p21 ras genes. Cancer Invest 2: 109-123, 1984.

17. Nikiforova MN, Lynch RA and Biddinger PW: RAS point mutations and PAX8-PPAR gamma rearrangement in thyroid tumors: evidence for distinct molecular pathways in thyroid follicular carcinoma. J Clin Endocrinol Metab 88: 2318-2326, 2003.

18. Taparowsky E, Shimizu K and Goldfarb M: Structure and activation of the human N-ras gene. Cell 34: 581-586, 1983.

19. Ichikawa Y, Nishida M and Suzuki H: Suppression of metastasis of rat prostatic cancer by introducing human chromosome 8 . Cancer Res 54: 2299-2302, 1994.

20. Suzuki M, Saito S and Saga Y: Mutation of K-RAS protooncogene and loss of heterozygosity on 6q27 in serous and mucinous ovarian carcinomas. Cancer Genet Cytogenet 118: 132-135, 2000.

21. Shih IeM and Kurman RJ: Molecular pathogenesis of ovarian borderline tumors: new insights and old challenges. Clin Cancer Res 11: 7273-7279, 2005.

22. Mammas IN, Zafiropoulos A and Spandidos DA: Involvement of the ras genes in female genital tract cancer (review). Int $\mathbf{J}$ Oncol 26: 1241-1255, 2005.

23. Zhang X, Vincent P and McHugh M: BAY 43-9006 exhibits broad spectrum oral antitumor activity and targets the RAF/ MEK/ERK pathway and receptor tyrosine kinases involved in tumor progression and angiogenesis. Cancer Res 64: 7099-7109, 2004.

24. Wilhelm SM, Carter C and Tang L: Proteasome inhibition and its clinical prospects in the treatment of hematologic and solid malignancies. Cancer 104: 1794-1807, 2005.

25. Hendrix MJC, Seftor EA and Chu YW: Role of intermediate filaments in migration, invasion and metastasis. Cancer Metastasis Rev 15: 507-525, 1996. 